\title{
ARTICLE OPEN Gene expression signatures of human cell and tissue longevity
}

\author{
Inge Seim ${ }^{1,2}$, Siming $\mathrm{Ma}^{1}$ and Vadim N Gladyshev ${ }^{1}$
}

Different cell types within the body exhibit substantial variation in the average time they live, ranging from days to the lifetime of the organism. The underlying mechanisms governing the diverse lifespan of different cell types are not well understood. To examine gene expression strategies that support the lifespan of different cell types within the human body, we obtained publicly available RNA-seq data sets and interrogated transcriptomes of 21 somatic cell types and tissues with reported cellular turnover, a bona fide estimate of lifespan, ranging from 2 days (monocytes) to a lifetime (neurons). Exceptionally long-lived neurons presented a gene expression profile of reduced protein metabolism, consistent with neuronal survival and similar to expression patterns induced by longevity interventions such as dietary restriction. Across different cell lineages, we identified a gene expression signature of human cell and tissue turnover. In particular, turnover showed a negative correlation with the energetically costly cell cycle and factors supporting genome stability, concomitant risk factors for aging-associated pathologies. In addition, the expression of p53 was negatively correlated with cellular turnover, suggesting that low p53 activity supports the longevity of post-mitotic cells with inherently low risk of developing cancer. Our results demonstrate the utility of comparative approaches in unveiling gene expression differences among cell lineages with diverse cell turnover within the same organism, providing insights into mechanisms that could regulate cell longevity.

npj Aging and Mechanisms of Disease (2016) 2, 16014; doi:10.1038/npjamd.2016.14; published online 7 July 2016

\section{INTRODUCTION}

Nature can achieve exceptional organismal longevity, > 100 years in the case of humans. However, there is substantial variation in 'cellular lifespan', which can be conceptualized as the turnover of individual cell lineages within an individual organism. ${ }^{1}$ Turnover is defined as a balance between cell proliferation and death that contributes to cell and tissue homeostasis. ${ }^{2}$ For example, the integrity of the heart and brain is largely maintained by cells with low turnover/long lifespan, while other organs and tissues, such as the outer layers of the skin and blood cells, rely on high cell turnover/short lifespan. ${ }^{3-5}$ Variation in cellular lifespan is also evident across lineages derived from the same germ layers formed during embryogenesis. For example, the ectoderm gives rise to both long-lived neurons ${ }^{4,6,7}$ and short-lived epidermal skin cells. ${ }^{8}$ Similarly, the mesoderm gives rise to long-lived skeletal muscle ${ }^{4}$ and heart muscle ${ }^{9}$ and short-lived monocytes, ${ }^{10,11}$ while the endoderm is the origin of long-lived thyrocytes (cells of the thyroid gland) ${ }^{12}$ and short-lived urinary bladder cells. ${ }^{13}$

How such diverse cell lineage lifespans are supported within a single organism is not clear, but it appears that differentiation shapes lineages through epigenetic changes to establish biological strategies that give rise to lifespans that support the best fitness for cells in their respective niche. As fitness is subject to trade-offs, different cell types will adjust their gene regulatory networks according to their lifespan. We are interested in gene expression signatures that support diverse biological strategies to achieve longevity. Prior work on species longevity can help inform strategies for tackling this research question. Species longevity is a product of evolution and is largely shaped by genetic and environmental factors. ${ }^{14}$ Comparative transcriptome studies of long-lived and short-lived mammals, and analyses that examined the longevity trait across a large group of mammals (tissue-by-tissue surveys, focusing on brain, liver and kidney), have revealed candidate longevity-associated processes. ${ }^{15,16}$ They provide gene expression signatures of longevity across mammals and may inform on interventions that mimic these changes, thereby potentially extending lifespan. It then follows that, in principle, comparative analyses of different cell types and tissues of a single organism may similarly reveal lifespan-promoting genes and pathways. Such analyses across cell types would be conceptually similar, yet orthogonal, to the analysis across species. Publicly available transcriptome data sets (for example, RNA-seq) generated by consortia, such as the Human Protein Atlas (HPA), ${ }^{17}$ Encyclopedia of DNA Elements (ENCODE), ${ }^{18}$ Functional Annotation Of Mammalian genome (FANTOM) ${ }^{19}$ and the Genotype-Tissue Expression (GTEx) project, ${ }^{20}$ are now available. They offer an opportunity to understand how gene expression programs are related to cellular turnover, as a proxy for cellular lifespan. Here we examined transcriptomes of 21 somatic cells and tissues to assess the utility of comparative gene expression methods for the identification of longevity-associated gene signatures.

\section{RESULTS}

We interrogated publicly available transcriptomes (paired-end RNA-seq reads) of 21 human cell types and tissues, comprising 153 individual samples, with a mean age of 56 years (Table 1; details in Supplementary Table S1). Their turnover rates (an estimate of cell lifespan ${ }^{4}$ ) varied from 2 (monocytes) to 32,850 (neurons) days, with all three germ layers giving rise to both short-lived and

\footnotetext{
${ }^{1}$ Division of Genetics, Department of Medicine, Brigham and Women's Hospital and Harvard Medical School, Boston, MA, USA.

Correspondence: VN Gladyshev (vgladyshev@rics.bwh.harvard.edu)

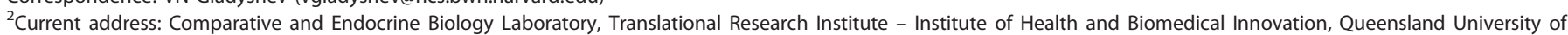
Technology, Woolloongabba, QLD 4102, Australia.

Received 21 August 2015; revised 8 March 2016; accepted 30 March 2016
} 
long-lived cell lineages. Biological replicates showed Pearson's correlation coefficients above 0.90, indicating reproducibility of the gene expression data (Supplementary Table S2; Supplementary Figure S1). Gene expression patterns were first analyzed by Principal Component Analysis (PCA) (Figure 1a), which revealed that the first three Principal Components ( $P C s$ ) accounted for $\sim 60 \%$ of gene expression variation. The cells and tissues formed several clusters, suggesting related biological functions for these clusters. For example, the gastrointestinal tissues, esophagus, rectum and colon all grouped together, and hematopoietic tissues (bone marrow and spleen) and monocytes also clustered. Because transcriptomes of functionally related cell types often exhibit substantial hierarchical structure, ${ }^{21,22}$ a

Table 1. Summary of human cells and tissues used in the study

\begin{tabular}{llc}
\hline Tissue/cell type & Germ layer & Estimated turnover (days) \\
\hline Adipose tissue & Mesoderm & 2,448 \\
Adrenal gland & Ectoderm & 455 \\
Bone marrow & Mesoderm & 3.2 \\
$\left(\right.$ CD14 ${ }^{+}$) monocytes & Mesoderm & 2 \\
Colon & Endoderm & 3.5 \\
Endometrium & Mesoderm & 13 \\
Esophagus & Endoderm & 10 \\
Heart muscle & Mesoderm & 25,300 \\
Keratinocytes (skin epidermis) & Ectoderm & 64 \\
Kidney & Mesoderm & 270 \\
Liver & Endoderm & 327 \\
Lung & Endoderm & 200 \\
Neuron (neocortex) & Ectoderm & 32,850 \\
Osteoblasts (bone) & Mesoderm & 8.3 \\
Rectum & Endoderm & 3.5 \\
Salivary gland & Ectoderm & 60 \\
Skeletal muscle & Mesoderm & 5,510 \\
Smooth muscle & Mesoderm & 67.5 \\
Spleen & Mesoderm & 7.8 \\
Thyroid gland & Endoderm & 3,180 \\
Urinary bladder & Endoderm & 49 \\
\hline See Supplementary Table S1 for further details. & \\
\hline
\end{tabular}

a

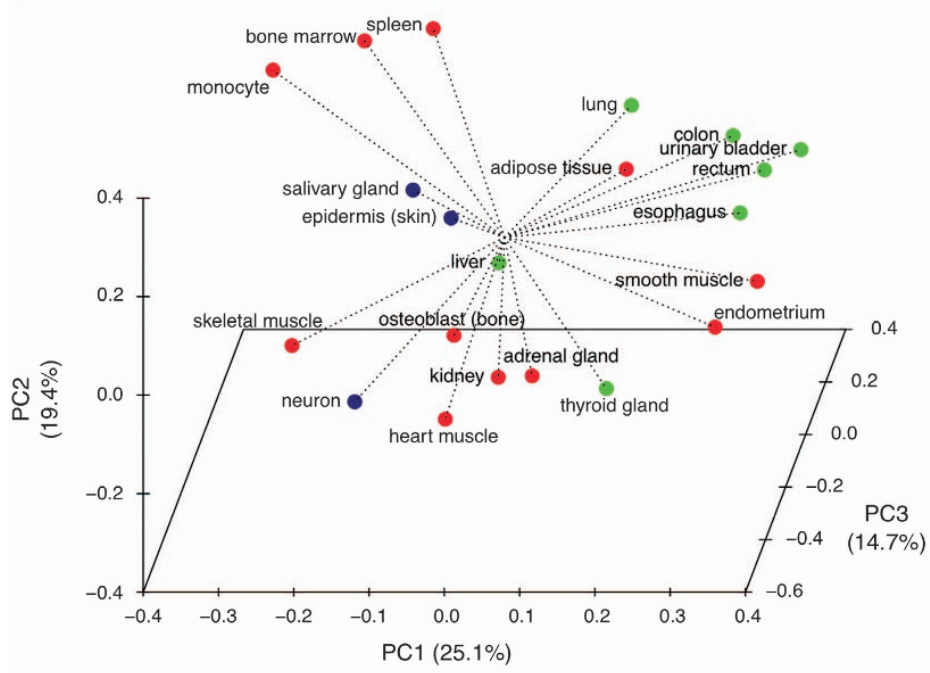

neighbor-joining gene expression tree was generated based on mean gene expression levels (Figure 1b). Similar to the PCA results, bone marrow and spleen clustered with monocytes, while skeletal muscle and heart muscle grouped together and were distinct from smooth muscle. Although the PCA and gene expression tree correlated with the broad functional features of the cells and tissues, the clustering did not strictly follow germ layer origin. In agreement, recent data demonstrate that the regulatory DNA landscape (epigenome), but not gene expression (transcriptome), correlate with cell lineage relationships, including germ layer origin. ${ }^{23-25}$ Thus, for any given cell type, e.g., a neuron, epigenetic marks reflect both the prior (e.g., state in the germ layer and derived cell lineages) and present regulatory landscapes. $^{24}$

Differential gene expression of cell and tissue groups

We selected several lineage groups and individual cells and tissues and compared each of them against all other cells and tissues in order to identify associated specific expression patterns (Supplementary Tables S3 and S4; Supplementary Results and Discussion). The results for heart (muscle), thyroid gland, hematopoietic tissues and monocytes are presented in Supplementary Figure S2. In heart and skeletal muscle, 455 out of 12,044 genes were differentially expressed (phylogenetic analysis of variance (ANOVA) $P$ value $\leqslant 0.01$ ) compared with other cells and tissues (Figure 2a). Approximately 44\% of these genes were associated with the tricarboxylic acid (TCA) cycle and respiration, in agreement with the metabolic organization and energy sources of these tissues. ${ }^{26}$

Neurons, which are critical for cognitive and motor functions, have cell lifespans that likely exceed the lifespan of the organism. ${ }^{7,27}$ Comparing neurons to shorter-lived cells and tissues is conceptually similar to comparing gene expression of long-lived mammals to related short-lived species, e.g., examining African mole rats against other rodents. ${ }^{15}$ Accordingly, neurons should possess a gene expression signature associated with low turnover/ long lifespan, in addition to the patterns indicative of neuronal function. Out of 12,044 genes 1,438 were differentially expressed in neurons ( $P \leqslant 0.01$; Figure $2 \mathrm{~b}$; Supplementary Table S3) and gene

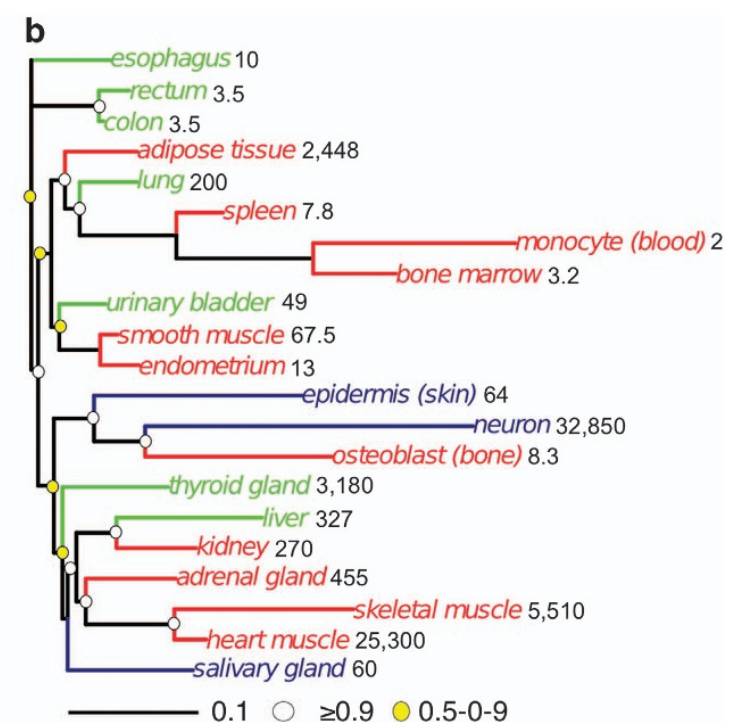

Figure 1. Clustering of gene expression from diverse human cells and tissues. (a) Representations of gene expression in Principal Component (PC) space. Values in parenthesis indicate the percentage of variance explained by each of the PCs. Ostensible germ layer origin is indicated (red: mesoderm; blue: ectoderm, green: endoderm). (b) Gene expression tree. Branches are colored according to germ layer origin (as in a). Estimated cell and tissue turnover ('cellular lifespan'; in days) is shown next to cell and tissue names. The tree was generated by neighborjoining (NJ) method (bootstrap $=1,000,000$ ). Bootstrap values are indicated by circles: white $\geqslant 0.9$; yellow $\leqslant 0.9$. 


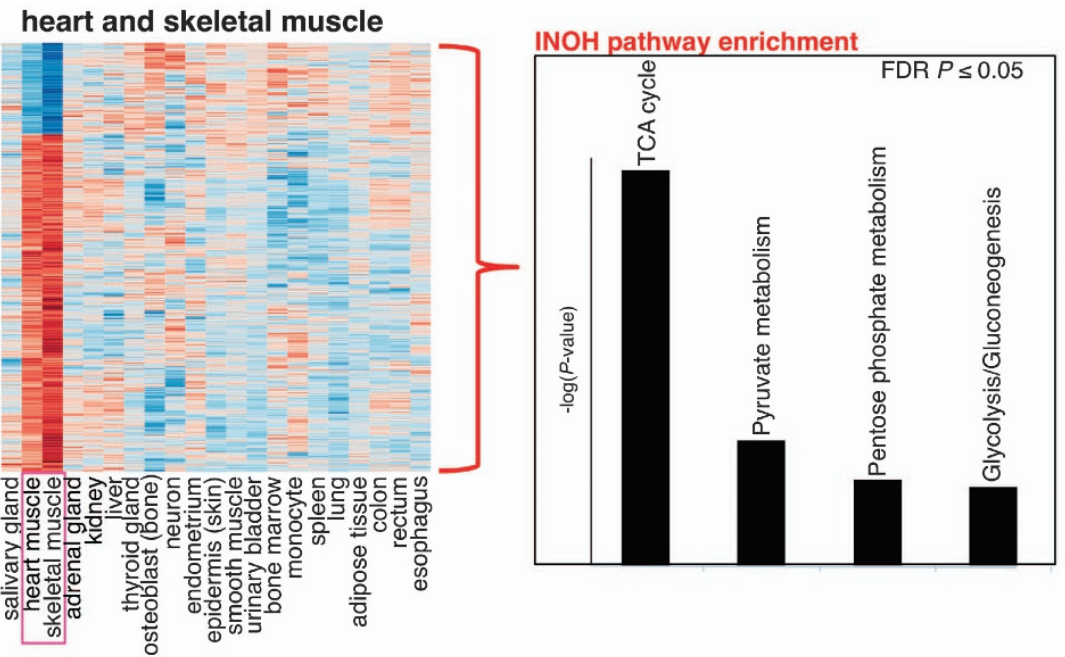

b

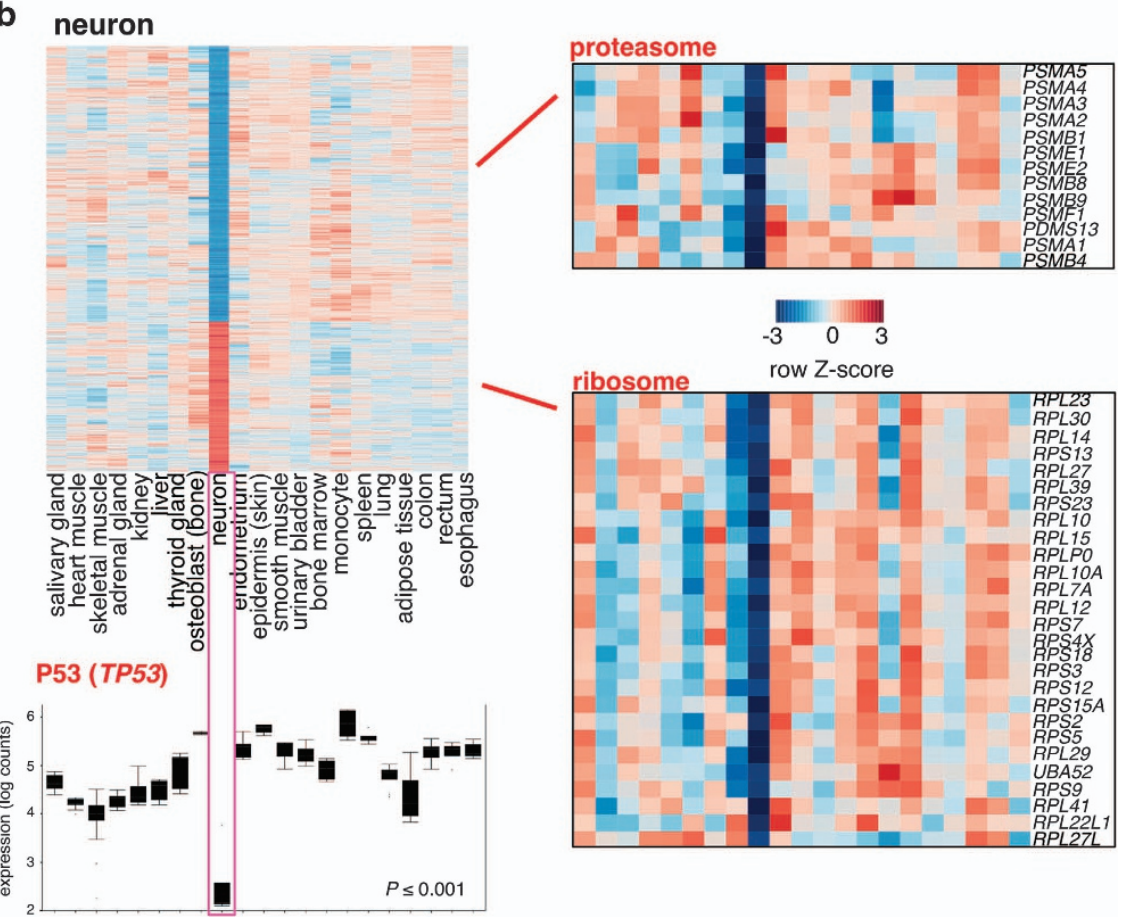

Figure 2. Genes differentially expressed between selected cell lineages and tissues. (a) Heart and skeletal muscle. Left panel, heat map of genes differentially expressed in heart and skeletal muscle relative to the other cells and tissues (standardized expression level; red: high expression; blue: low expression). Right panel, significantly enriched (FDR $P \leqslant 0.05$ ) pathways. (b) Neuron. Left panel, heat map of genes differentially expressed in neurons, colored and annotated as in a. Right panel, significantly enriched (FDR $P \leqslant 0.05$ ) pathways 'ribosome' and 'proteasome'. FDR P denotes false discovery rate-adjusted $P$ value. Lower panel, box plot showing p53 (TP53) expression. Error bars indicate s.e.m.

set enrichment analysis showed enrichment for functions associated with lysosomes, proteasomes, ribosomal proteins and apoptosis (Supplementary Table S3). Neurons presented with reduced expression of 27 ribosomal proteins and multiple $20 \mathrm{~S}$ proteasome subunit genes (Figure 2b), consistent with distinct protein metabolism required to fine-tune self-renewal and synaptic plasticity. ${ }^{28}$ This group of genes was not correlated with cell and tissue turnover (see section below), suggesting that this expression pattern is unique to long-lived neurons. Reduced protein metabolism, which may be induced by dietary restriction and other interventions, is known to associate with extended lifespan in a number of model organisms. ${ }^{29,30}$ Furthermore, expression of the tumor suppressor p53 (TP53) was significantly reduced $(P \leqslant 0.001)$ in neurons, where it was expressed at a level
5-30 times lower than that in the other cells and tissues (Figure 2b). Reduced p53 expression is associated with a concomitant reduction in cell cycle-related proteins in neurons following their terminal differentiation from neuroblasts. ${ }^{31}$

Gene expression patterns of cell and tissue turnover We identified genes whose expression correlated with cell and tissue turnover. Available turnover times for a number of tissues and cell types (in days) ${ }^{3}$ were supplemented with estimates from the literature and used as a bona fide measure of lifespan ('lifespan trait'). We applied generalized least squares regression, ${ }^{32}$ tested different evolutionary models and selected the best fit model by maximum likelihood (see Extended Experimental Procedures). 
Two hundred eight out of 12,044 genes showed significant correlation with turnover at a false discovery rate (Q-value) of 0.05 , with $75 \%$ (155 genes) in negative correlation and $25 \%$ (53 genes) in positive correlation (Supplementary Table S5). Notable genes with a positive correlation included the complex SNRPN-SNURF locus, which gives rise to a number of proteins and short non-coding RNAs (Supplementary Figure S3; Supplementary Results and Discussion). We visualized the protein-protein interaction network represented by these 208 genes, revealing significant enrichment (FDR $P \leqslant 0.05)$ for genes involved in cell cycle, immune signaling (NF-KB) and p53 signaling (Figure 3a,b and Supplementary Tables S6 and S7). In our data set, hematopoietic tissues (bone marrow and spleen) and monocytes constituted the samples with the shortest turnover. Removal of these data points in the regression analysis retained the 'turnover signature', with the overlapping gene set comprising critical cell cycle and apoptosis associated genes, such as CHEK1, CHEK2, MKI67, FOXM1, TP53 and BCL10, while a correlation with immune signaling-associated genes was lost (Table 2 and Supplementary Table S8).

Negative correlation between cell cycle and associated genome integrity pathways and cell and tissue turnover

Gene ontology, KEGG pathway analysis and manual interrogation of genes correlating with turnover revealed that $30-40 \%$ of these (Supplementary Tables S5 and S8) have roles in the cell cycle, a highly complex multi-step process. They spanned all phases of the cell cycle, with the majority of the genes showing decreased a
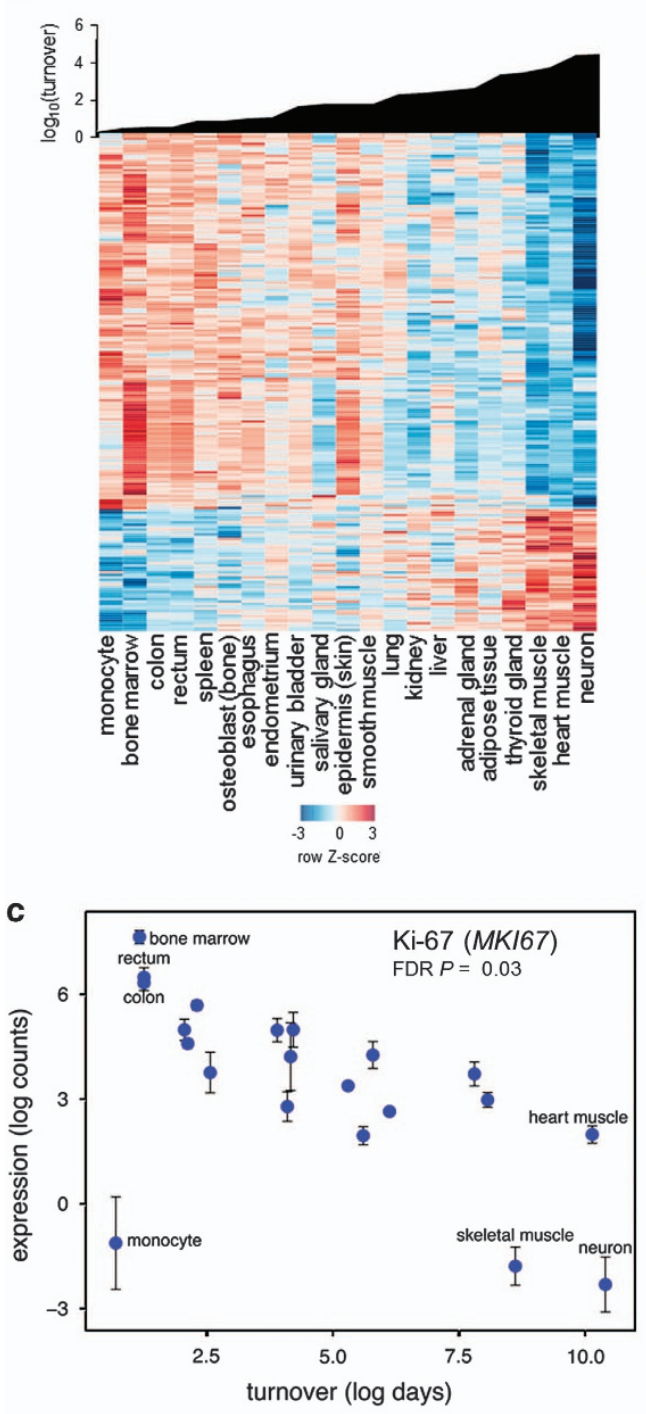

b
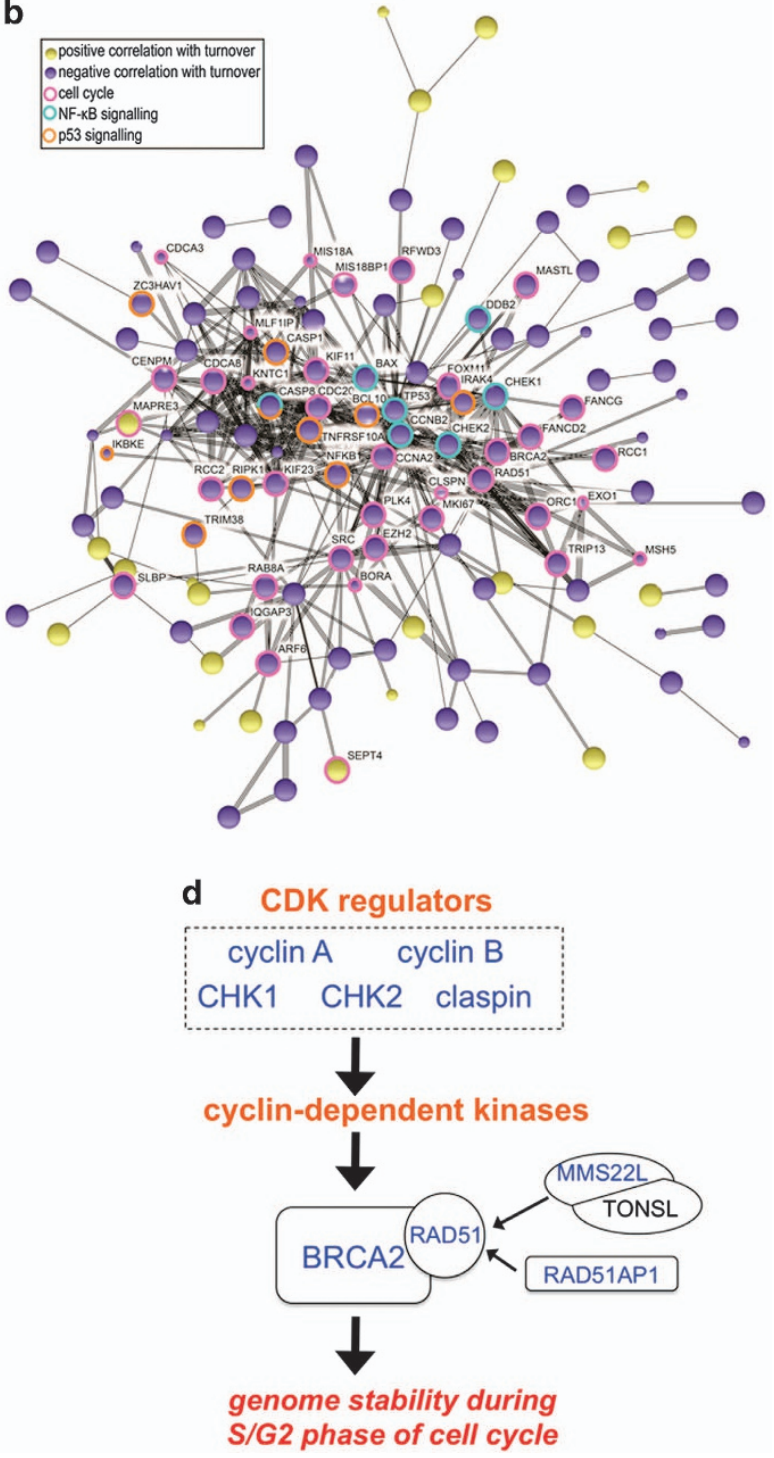

Figure 3. Overview of genes correlating with cellular turnover. (a) Heat map analysis. Upper panel, cellular turnover of the cells and tissues (in ascending order). Lower panel, heat map displaying gene expression of genes correlating with turnover (standardized expression levels; red: high expression; blue: low expression). (b) Network analysis. Protein-protein interaction network of genes correlating with turnover. The interaction network was created by interrogating the STRING database (evidence view). Lines (strings) indicate protein interactions. Proteins without interacting partners were omitted. Selected pathways are indicated by colored rings. (c) Expression of the cell proliferation marker Ki-67 (MKI67) correlates negatively with turnover. Error bars indicate standard error of the mean. FDR $P$ denotes false discovery rate $P$ value. (d) Genes associated with RAD51-mediated genome stability control in the replication phase of the cell cycle. Names highlighted in blue showed negative correlation with turnover. CDK: cyclin-dependent kinase; CCNA2: cyclin A2; CCNB2: cyclin B2; CHEK1: checkpoint kinase 1, CHK1; CHEK2: checkpoint kinase 2, CHK2; CLSPN: claspin; BRCA2: breast cancer 2, early onset; RAD51: RAD51 recombinase; RAD51AP1: RAD51 associated protein 1; MMS22L: MMS22-like, DNA repair protein; TONSL: tonsoku-like, DNA repair protein. 
Table 2. Overlap of genes correlating with turnover before and after removal of immune system-associated cells and tissues (monocytes, bone marrow and spleen)

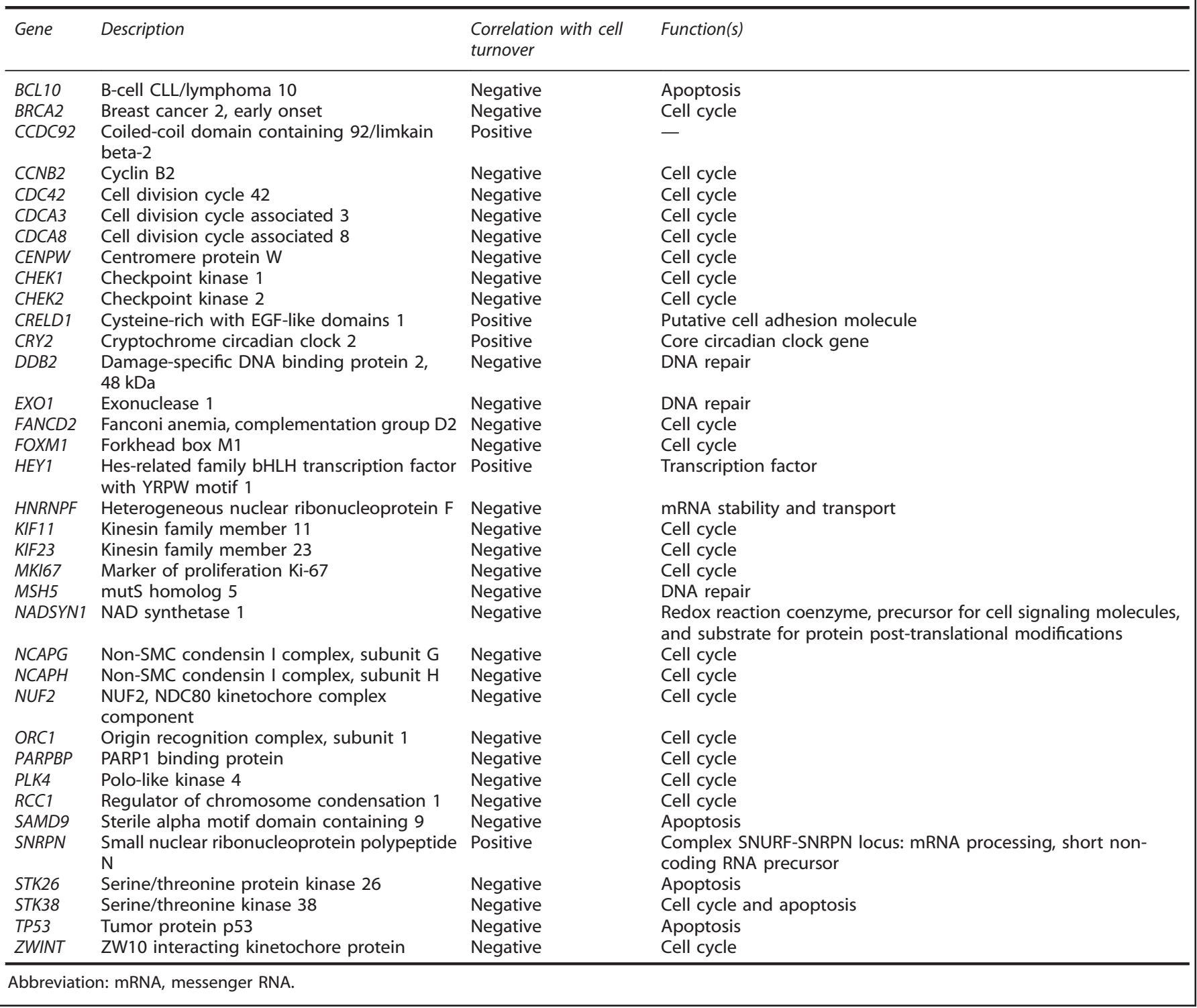

expression associated with increased turnover. As expected, the classic DNA replication marker, Ki-67 (MKI67), which is only expressed during the cell cycle, ${ }^{33}$ was present at high levels in rapidly proliferating tissues such as bone marrow, rectum and colon. It was expressed at very low levels in monocytes (the majority of which do not proliferate) and in post-mitotic skeletal muscle and neurons (most of which are incapable of cell division; Figure 3c). Ki-67 was expressed at a moderate level in heart muscle, which can proliferate at a limited rate, ${ }^{9}$ and where Ki-67 may promote postnatal cardiac remodeling. ${ }^{34}$

A negative correlation between the cell cycle machinery and cell and tissue turnover is not surprising, as proliferative homeostasis, a balance between cell growth and death, is essential for normal turnover (as occurs in epidermal cells, for example). In contrast, terminally differentiated cells comprising heart muscle, skeletal muscle and neurons, have permanently or largely exited the cell cycle. They are, therefore, expected to express low levels of genes related to cell cycle checkpoints and the maintenance of replication fidelity. We noted that a number of genes essential for genome stability during the premitotic phase
(G2), a process tightly linked to tumor development, ${ }^{35}$ were negatively correlated with turnover (Figure $3 \mathrm{~d}$ ). This included regulators of cyclin-dependent kinases, such as cyclin $A$ (CCNA2) and $B(C C N 2 B)$, checkpoint kinase 1 (CHEK1) and its regulator claspin (CLSPN), and CHEK2, which together with breast cancer 2 early onset (BRCA2), RAD51 recombinase (RAD51), its enhancer RAD51AP1 (RAD51AP1), and MMS22-like DNA repair protein (MMS22L) promote genome stability. ${ }^{35}$ These data support recent work showing that RAD51 and BRCA2 are major facilitators of genome integrity in proliferating cells. ${ }^{35-37}$ Genes in the p53 pathway can halt progression of the cell cycle (induce senescence), or inhibit apoptosis in proliferating cells, and blocking apoptosis is crucial for the survival of differentiated post-mitotic cells. ${ }^{38}$ In agreement with a reduction in the involvement of cell cycle in longer-lived cells and tissues, multiple p53 pathway associated genes, including p53 itself (TP53), were negatively correlated with turnover (Supplementary Figure S4; Supplementary Tables S5 and S8). They encompassed several checkpoint kinases (CHEK1 and CHEK2), BCL2-associated X protein 
(BAX), which activates apoptosis, and ASC/TMS1 (PYCARD), which when downregulated inhibits $B A X$ translocation to mitochondria.

To further investigate the connection between gene expression and turnover, we turned to the library of integrated networkbased cellular signatures (LINCS) to identify perturbations (gene overexpression and knockdown) that produce a similar overall gene expression profile to our regression analysis. We found that the most similar profiles came from genes associated with cell cycle control and associated DNA repair (Supplementary Table $\mathrm{S} 11)$. This included overexpression of the major cell cycle inhibitors p18 (also known as INK4C; CDKN2C), p21 (CDKN1A) and p27 (CDKN1B), in agreement with the negative correlation between downstream genes and turnover in our data set.

No correlation between oxidative phosphorylation gene expression and cell and tissue turnover

It is clear that mitochondrial dysfunction is a hallmark of aging. Properly functioning mitochondria are essential for energy production and cell survival, and hence, are crucial for longevity and resistance to age-related disease. ${ }^{39}$ We calculated pair-wise correlations of the expression of 97 nuclear-encoded genes associated with oxidative phosphorylation (OXPHOS) ${ }^{40}$ across the data set, revealing a strong correlation across all samples with the exception of heart muscle and skeletal muscle (Figure 4a). As shown in Figure 4b, expression of individual OXPHOS genes varied by tissue, in agreement with a previous microarray study, ${ }^{40}$ but there was no overall correlation with cell and tissue turnover.

\section{DISCUSSION}

Several interrelated evolutionary and mechanistic theories have been proposed that provide insights into the evolution of lifespan and suggest the involvement of a large armamentarium of genes. ${ }^{41,42}$ In this study, we employed gene expression data from 21 somatic cell types and tissues and sought to identify genes and pathways associated with cell and tissue turnover, to our knowledge the current best estimate of cellular lifespan, ${ }^{1}$ in one of the longest-lived mammals, human. We first tested for differential expression in selected groups of cells and tissues, revealing expression patterns that fit well with expected biological functions, including the TCA cycle and respiration in cardiac and skeletal muscle; immune function genes in bone marrow, monocyte and spleen, and reduced protein metabolism in neurons.

The major insights of our study centered on the relationship between gene expression and cell and tissue turnover. Multiple genes taking part in the energetically expensive cell cycle and associated repair (genome stability) were negatively correlated with turnover. By interrogating the Broad Institute's Library of Integrated Cellular Signatures resource, we found that very similar gene expression patterns can be achieved by targeted overexpression and knockdown of single genes, suggesting that gene expression patterns associated with cell and tissue turnover, and by proxy cell lifespan, may in principle be achieved by genetic, pharmacological and perhaps dietary interventions. The observation that turnover negatively correlates with cell cycle genes may seem tautological. Indeed, one would expect tissue or cell populations with high turnover to exhibit more cells in the cell cycle and, consequently, high expression of cell cycle and associated genes. However, it is recognized that the rate of aging and longevity is indeed fine tuned by the balance of cell division and death (i.e., cell turnover). ${ }^{2}$ Interestingly, a recent study examined the transcriptional response to long-term calorie restriction in humans, revealing that calorie restriction shifts cellular metabolism of skeletal muscle from proliferation to maintenance and repair. ${ }^{43}$ The concomitant reduction in cell cycle gene expression presumably results in a 'younger' transcriptional signature that contributes to the lifespanextending properties of calorie restriction.

Expression of p53 (TP53), often referred to as the 'guardian of the genome', was negatively correlated with cell and tissue turnover; with particularly low levels of expression in long-lived neurons. Evidence is emerging that p53 has an evolutionarily ancient lifespan-regulating function, in addition to its role as tumor suppressor. It is appreciated that p53 promotes organismal longevity by preventing survival of abnormal cells; however, several investigators have speculated that p53 protects against
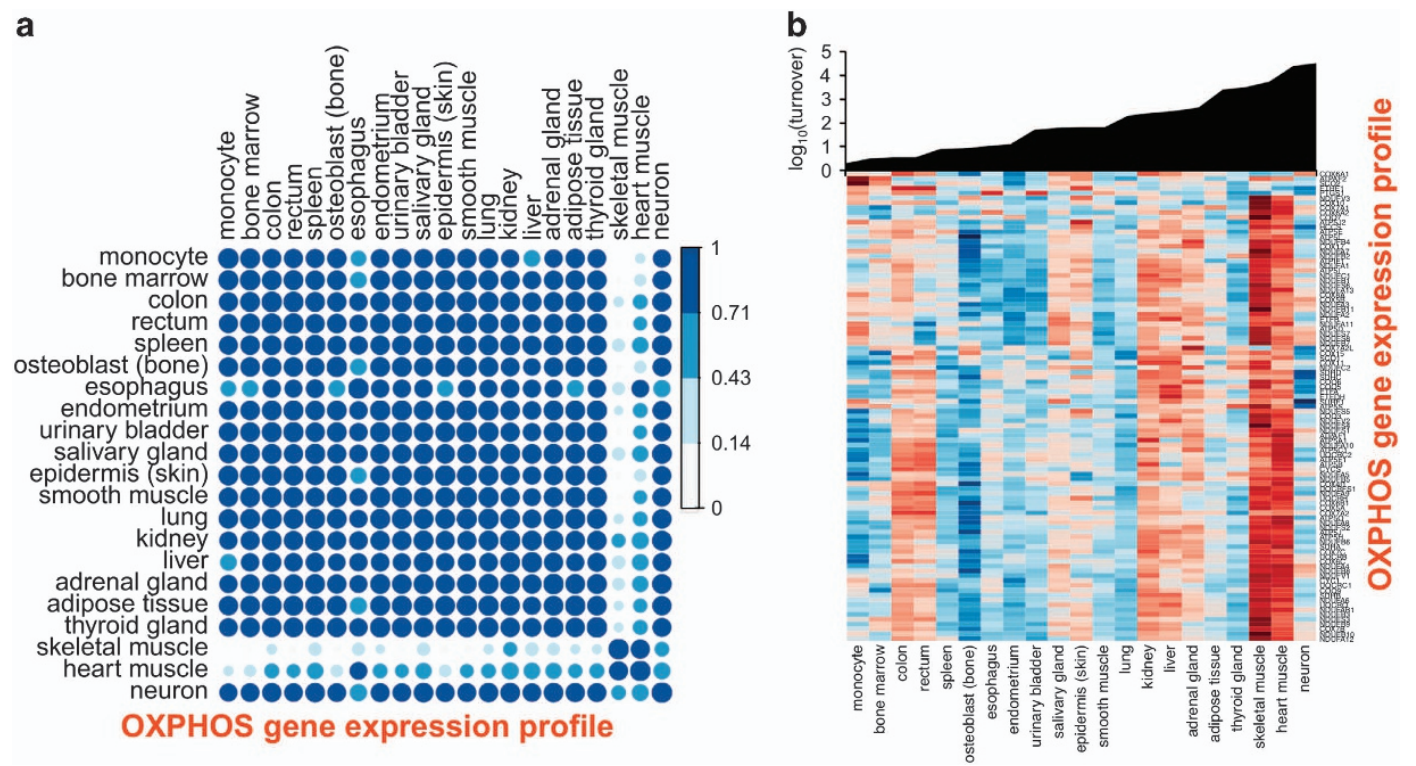

Figure 4. Expression of nuclear-encoded oxidative phosphorylation (OXPHOS) genes in 21 human somatic cells and tissues. (a) Pairwise correlation matrix of 97 OXPHOS genes. Darker blue shading indicating stronger positive correlation. Blue corresponds to a correlation of $\sim 1$ and white to $\sim 0$. (b) Upper panel, cellular turnover of the cells and tissues (in ascending order). Lower panel, heat map displaying gene expression of individual OXPHOS genes. Scaled $\log _{2}$ transformed normalized counts (Z-score) are plotted in blue-red color, with red indicating high expression and blue indicating low expression. 
cancer in proliferating cells at the cost of accelerated aging. 3,44,45 Thus, in non-dividing cells p53 may, in effect, reduce lifespan. It was found that suppression of TP53 orthologs in animal models such as the mouse and fruit fly can extend organismal lifespan (reviewed in ref. 46). Dominant-negative Drosophila p53 (Dmp53) significantly extends organismal lifespan when expressed in adult neurons, but not other tissues (such as muscle) via insulin/ insulin-like signaling (IIS) and TOR (target of rapamycin) pathways. ${ }^{47,48}$ Several p53 (TP53) retrogenes have been recently reported in the elephant genome, ${ }^{49}$ however, while elephant lymphocytes and fibroblasts show an increased response to DNA damage compared with human cells, ${ }^{49}$ it is currently not known how many of these retrogenes are actively translated and exhibit p53 function, nor whether the expression of p53 is appropriately activated and/or elevated in all elephant cells. Thus, the elephant's resistance to cancer may stem from other mechanisms and it would be of interest to examine the expression of TP53 in elephant neurons and other cell types. Interestingly, the cancer-resistant long-lived blind mole rat, Spalax, has evolved an enhanced necrotic and reduced apoptotic defense (via a dominant negative form of p53) against cancer, possibly to adapt to an oxygen-poor underground environment, which would normally result in extensive p53-mediated cell death. ${ }^{50,51}$ Whether Spalax p53 contributes to the longevity of this cancer-resistant rodent is not known, but is an exciting possibility under the hypothesis that reduced p53 activity exerts beneficial effects on cellular lifespan if tumor formation is avoided. Taken together, we speculate that very low levels of endogenous p53 contribute to the exceptional lifespan of cells and tissues with low turnover, such as neuron, heart muscle and skeletal muscle, and perhaps also organismal longevity.

It is now appreciated that expression of OXPHOS genes decreases with age in diverse cells and tissues in species ranging from nematode to human. ${ }^{39}$ However, the link between mitochondrial homeostasis and lifespan is currently enigmatic. ${ }^{39}$ Interestingly, lifespans of different strains of the single-celled yeast is associated with upregulation of OXPHOS genes. ${ }^{52}$ In our data set of 21 human cells and tissues, expression of individual OXPHOS genes, and more generally metabolic genes, did not correlate with cell turnover. Thus, we propose that while cells and tissues share a gene expression signature manifested as reduced mitochondrial function with age, the overall integrity of mitochondria in long-lived human cell types is achieved by distinct gene expression strategies.

Our study has several limitations. Future studies should attempt to more accurately determine the turnover of cells and cell populations (tissues) in the body, and sequence the transcriptomes of additional long-lived post-mitotic cells, such as osteocytes. ${ }^{53}$ Single-cell RNA sequencing is rapidly evolving ${ }^{54}$ and would greatly advance the study of cell turnover, especially as it would avoid the analysis of organs that are composed of heterogeneous cell types. Multiple cells and tissues from the same individual are also becoming available. ${ }^{55}$ Finally, the contribution of epigenetics ${ }^{56,57}$ and long-lived proteins ${ }^{58,59}$ to cell and tissue turnover is largely unknown but likely important. Owing to a lack of a matching set of samples and limited number of biological replicates, we did not interrogate other species, such as the mouse, for turnover-associated genes. It is currently unknown whether individual genes identified through our procedure would overlap among species, ${ }^{60}$ but is an important question for future studies. Although some common features may be observed, human cell types may also harbor signatures quite distinct from other animals, including other primates, since humans are one of the most exceptionally long-lived species. ${ }^{61}$

Overall, our analysis, employing cellular turnover, as a proxy of lifespan, is a first step to a molecular understanding of cell and tissue longevity. We reveal a gene signature of exceptionally long-lived post-mitotic neurons, and genes and pathways that correlate with turnover across 21 somatic cells and tissues. The data suggest that human cell lineages utilize both common and lineage-specific strategies to alter their lifespan. This new perspective should provide further impetus to the study of the lifespan trait (longevity) and the aging process.

\section{MATERIALS AND METHODS}

See Supplementary Information for detailed methods.

\section{Biological samples}

Our analysis was restricted to 21 adult somatic cells and tissues with more than three biological replicates and bona fide lifespan estimates (cell turnover in days) derived from a recent comprehensive review ${ }^{3}$ and the additional data collected through primary literature searches (see Supplementary Experimental Procedures).

\section{Transcriptome data relationship inference}

The relationship of publicly available transcriptomes (RNA-seq data) from the 21 cells and tissues was investigated by principal component analysis and a gene expression tree (see Supplementary Experimental Procedures).

Identification of genes differentially expressed between cell and tissue groups

Differentially expressed genes in a particular group (e.g., heart and skeletal muscle) were identified by ANOVA, taking into account the hierarchical relationship between samples in our data set (tissue autocorrelation by 'phylogenetic ANOVA'). See Supplementary Experimental Procedures for further details.

Identification of genes correlating with cell and tissue turnover To identify genes correlating with cell turnover (a bona fide lifespan estimate) we employed generalized least squares regression (Supplementary Experimental Procedures).

\section{ACKNOWLEDGEMENTS}

We acknowledge financial support from NIH (AG047745 and AG021518).

\section{CONTRIBUTIONS}

I.S. performed the analyses. V.N.G. supervised the study. All authors contributed to data interpretation. I.S. and V.N.G. wrote the paper, with significant contributions from S.M.

\section{COMPETING INTERESTS}

The authors declare no conflict of interest.

\section{REFERENCES}

1. Spalding, K. L. et al. Dynamics of fat cell turnover in humans. Nature 453 783-787 (2008).

2. Pellettieri, J. \& Sanchez Alvarado, A. Cell turnover and adult tissue homeostasis: from humans to planarians. Annu. Rev. Genet. 41, 83-105 (2007).

3. Richardson, R. B., Allan, D. S. \& Le, Y. Greater organ involution in highly proliferative tissues associated with the early onset and acceleration of ageing in humans. Exp. Gerontol. 55, 80-91 (2014).

4. Spalding, K. L., Bhardwaj, R. D., Buchholz, B. A., Druid, H. \& Frisen, J. Retrospective birth dating of cells in humans. Cell 122, 133-143 (2005).

5. Bergmann, O. et al. Dynamics of cell generation and turnover in the human heart Cell 161, 1566-1575 (2015).

6. Huttner, H. B. et al. The age and genomic integrity of neurons after cortical stroke in humans. Nat. Neurosci. 17, 801-803 (2014).

7. Magrassi, L., Leto, K. \& Rossi, F. Lifespan of neurons is uncoupled from organismal lifespan. Proc. Natl Acad. Sci. USA 110, 4374-4379 (2013).

8. Halprin, K. M. Epidermal 'turnover time'--a re-examination. Br. J. Dermatol. 86, 14-19 (1972)

9. Bergmann, O. et al. Evidence for cardiomyocyte renewal in humans. Science 324, 98-102 (2009). 
10. Fahy, R. J., Doseff, A. I. \& Wewers, M. D. Spontaneous human monocyte apoptosis utilizes a caspase-3-dependent pathway that is blocked by endotoxin and is independent of caspase-1. J. Immunol. 163, 1755-1762 (1999).

11. Mangan, D. F. \& Wahl, S. M. Differential regulation of human monocyte programmed cell death (apoptosis) by chemotactic factors and pro-inflammatory cytokines. J. Immunol. 147, 3408-3412 (1991).

12. Coclet, J., Foureau, F., Ketelbant, P., Galand, P. \& Dumont, J. E. Cell population kinetics in dog and human adult thyroid. Clin. Endocrinol. (Oxf) 31, 655-665 (1989).

13. Leblond, C. P. \& Walker, B. E. Renewal of cell populations. Physiol. Rev. 36, 255-276 (1956).

14. Govindaraju, D., Atzmon, G. \& Barzilai, N. Genetics, lifestyle and longevity: Lessons from centenarians. Appl. Transl. Genomics 4, 23-32 (2015).

15. Fang, X. et al. Adaptations to a subterranean environment and longevity revealed by the analysis of mole rat genomes. Cell Rep. 8, 1354-1364 (2014).

16. Fushan, A. A. et al. Gene expression defines natural changes in mammalian lifespan. Aging Cell 14, 352-365 (2015).

17. Uhlen, M. et al. Proteomics. Tissue-based map of the human proteome. Science 347, 1260419 (2015).

18. ENCODE Project Consortium. An integrated encyclopedia of DNA elements in the human genome. Nature 489, 57-74 (2012)

19. Fantom Consortium. A promoter-level mammalian expression atlas. Nature 507, 462-470 (2014).

20. Mele, M. et al. Human genomics. The human transcriptome across tissues and individuals. Science 348, 660-665 (2015).

21. Liang, C., Consortium, F., Forrest, A. R. \& Wagner, G. P. The statistical geometry of transcriptome divergence in cell-type evolution and cancer. Nat. Commun. 6, 6066 (2015).

22. Kin, K., Nnamani, M. C., Lynch, V. J., Michaelides, E. \& Wagner, G. P. Cell-type phylogenetics and the origin of endometrial stromal cells. Cell Rep. 10, 1398-1409 (2015).

23. Chen, H. et al. An integrative analysis of TFBS-clustered regions reveals new transcriptional regulation models on the accessible chromatin landscape. Sci. Rep. 5, 8465 (2015).

24. Stergachis, A. B. et al. Developmental fate and cellular maturity encoded in human regulatory DNA landscapes. Cell 154, 888-903 (2013).

25. Roadmap Epigenomics Consortium. Integrative analysis of 111 reference human epigenomes. Nature 518, 317-330 (2015).

26. Gibala, M. J., Young, M. E. \& Taegtmeyer, H. Anaplerosis of the citric acid cycle: role in energy metabolism of heart and skeletal muscle. Acta Physiol. Scand. 168, 657-665 (2000).

27. Herndon, L. A. et al. Stochastic and genetic factors influence tissue-specific decline in ageing C. elegans. Nature 419, 808-814 (2002).

28. Dong, C., Bach, S. V., Haynes, K. A. \& Hegde, A. N. Proteasome modulates positive and negative translational regulators in long-term synaptic plasticity. J. Neurosci. 34, 3171-3182 (2014).

29. Stout, G. J. et al. Insulin/IGF-1-mediated longevity is marked by reduced protein metabolism. Mol. Syst. Biol. 9, 679 (2013).

30. Mayer, C. \& Grummt, I. Ribosome biogenesis and cell growth: mTOR coordinates transcription by all three classes of nuclear RNA polymerases. Oncogene 25, 6384-6391 (2006).

31. Ferreira, A. \& Kosik, K. S. Accelerated neuronal differentiation induced by p53 suppression. J. Cell Sci. 109, 1509-1516 (1996).

32. Rohlf, F. J. A comment on phylogenetic correction. Evolution 60, 1509-1515 (2006).

33. Scholzen, T. \& Gerdes, J. The Ki-67 protein: from the known and the unknown. J. Cell. Physiol. 182, 311-322 (2000).

34. Ciulla, M. M. et al. Ki67 cytoplasmic expression: observations in normal tissue from heart atrial appendages of healthy rats. Cell Cycle 8, 2125 (2009).

35. Yata, K. et al. BRCA2 coordinates the activities of cell-cycle kinases to promote genome stability. Cell Rep. 7, 1547-1559 (2014).

36. Esashi, F. et al. CDK-dependent phosphorylation of BRCA2 as a regulatory mechanism for recombinational repair. Nature 434, 598-604 (2005).
37. O'Donnell, L. et al. The MMS22L-TONSL complex mediates recovery from replication stress and homologous recombination. Mol. Cell 40, 619-631 (2010).

38. Campisi, J. \& d'Adda di Fagagna, F. Cellular senescence: when bad things happen to good cells. Nat. Rev. Mol. Cell Biol. 8, 729-740 (2007).

39. Wang, Y. \& Hekimi, S. Mitochondrial dysfunction and longevity in animals: Untangling the knot. Science 350, 1204-1207 (2015).

40. Mootha, V. K. et al. Integrated analysis of protein composition, tissue diversity, and gene regulation in mouse mitochondria. Cell 115, 629-640 (2003).

41. Gladyshev, V. N. The free radical theory of aging is dead. Long live the damage theory!. Antioxid. Redox Signal. 20, 727-731 (2014).

42. Lopez-Otin, C., Blasco, M. A., Partridge, L., Serrano, M. \& Kroemer, G. The hallmarks of aging. Cell 153, 1194-1217 (2013).

43. Mercken, E. M. et al. Calorie restriction in humans inhibits the PI3K/AKT pathway and induces a younger transcription profile. Aging Cell 12, 645-651 (2013).

44. van Heemst, D. et al. Variation in the human TP53 gene affects old age survival and cancer mortality. Exp. Gerontol. 40, 11-15 (2005).

45. Rodier, F., Campisi, J. \& Bhaumik, D. Two faces of p53: aging and tumor suppression. Nucleic Acids Res. 35, 7475-7484 (2007).

46. Ukraintseva, S. V. et al. Trade-offs between cancer and other diseases: do they exist and influence longevity? Rejuvenation Res. 13, 387-396 (2010).

47. Bauer, J. H., Poon, P. C., Glatt-Deeley, H., Abrams, J. M. \& Helfand, S. L. Neuronal expression of p53 dominant-negative proteins in adult Drosophila melanogaster extends life span. Curr. Biol. 15, 2063-2068 (2005).

48. Bauer, J. H., Chang, C., Bae, G., Morris, S. N. \& Helfand, S. L. Dominant-negative Dmp53 extends life span through the dTOR pathway in D. melanogaster. Mech. Ageing Dev. 131, 193-201 (2010).

49. Abegglen, L. M. et al. Potential mechanisms for cancer resistance in elephants and comparative cellular response to DNA damage in humans. JAMA 314, 1850-1860 (2015)

50. Ashur-Fabian, O. et al. Evolution of p53 in hypoxia-stressed Spalax mimics human tumor mutation. Proc. Natl Acad. Sci. USA 101, 12236-12241 (2004).

51. Fang, X. et al. Genome-wide adaptive complexes to underground stresses in blind mole rats Spalax. Nat. Commun. 5, 3966 (2014).

52. Kaya, A. et al. Defining molecular basis for longevity traits in natural yeast isolates. npj Aging Mech. Dis. 1, 15001 (2015).

53. Knothe Tate, M. L., Adamson, J. R., Tami, A. E. \& Bauer, T. W. The osteocyte. Int. J. Biochem. Cell Biol. 36, 1-8 (2004).

54. Shapiro, E., Biezuner, T. \& Linnarsson, S. Single-cell sequencing-based technologies will revolutionize whole-organism science. Nat. Rev. Genet. 14, 618-630 (2013).

55. GTEx Consortium. Human genomics. The Genotype-Tissue Expression (GTEx) pilot analysis: multitissue gene regulation in humans. Science 348, 648-660 (2015).

56. Horvath, S. et al. Obesity accelerates epigenetic aging of human liver. Proc. Natl Acad. Sci. USA 111, 15538-15543 (2014).

57. Horvath, S. DNA methylation age of human tissues and cell types. Genome Biol. 14, R115 (2013)

58. Toyama, B. H. et al. Identification of long-lived proteins reveals exceptional stability of essential cellular structures. Cell 154, 971-982 (2013).

59. Savas, J. N., Toyama, B. H., Xu, T., Yates, J. R. 3rd \& Hetzer, M. W. Extremely long-lived nuclear pore proteins in the rat brain. Science 335, 942 (2012).

60. Khaitovich, P. et al. A neutral model of transcriptome evolution. PLoS Biol. 2, E132 (2004)

61. Pickering, A. M., Lehr, M. \& Miller, R. A. Lifespan of mice and primates correlates with immunoproteasome expression. J. Clin. Invest. 125, 2059-2068 (2015).

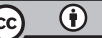

This work is licensed under a Creative Commons Attribution 4.0 International License. The images or other third party material in this article are included in the article's Creative Commons license, unless indicated otherwise in the credit line; if the material is not included under the Creative Commons license, users will need to obtain permission from the license holder to reproduce the material. To view a copy of this license, visit http://creativecommons.org/licenses/ by/4.0/

Supplementary Information accompanies the paper on the npj Aging and Mechanisms of Disease website (http://www.nature.com/npjamd) 